\title{
Cell-Based Gene Therapies and Stem Cells for Regeneration of Ischemic Tissues
}

\author{
Rosalinda Madonna ${ }^{1,2}$ and Raffaele De Caterina ${ }^{2,3}$ \\ ${ }^{1}$ Texas Heart Institute and University of Texas Medical School, Houston, \\ 2Institute of Cardiology, "G. d'Annunzio" University - Chieti, \\ "Fondazione "G. Monasterio" - Pisa, \\ ${ }^{1}$ Texas \\ 2,3Italy
}

\section{Introduction}

Coronary (CAD) and peripheral (PAD) artery disease are major causes of morbidity and mortality, requiring bypass surgery or angioplasty in approximately one million patients/year in the world (MERIT-HF Study Group, 1999). While collateral vessel formation as an alternative pathway for blood supply occurs in some of these patients, many do not form vascular networks adequate to compensate for the loss of the original blood supply (Hirsch et al., 2006). These patients might therefore benefit from stem cell transplantation therapies that would accelerate natural processes of postnatal collateral vessel formation, an approach referred to as therapeutic angiogenesis. On the other hand, recent seminal reports have indicated that the adult heart is self-healing and self-renewing. Specifically, these studies have demonstrated that there is a pool of resident cardiac stem cells (CSCs) that are clonogenic and multipotent and are capable of differentiating into new blood vessels or into new myocytes, and of cardiac progenitor cells (CPCs) (Marban, 2007). This suggests the possibility of using a therapeutic angiogenesis approach to complement other treatments (e.g., stem cell therapy) that facilitate myocardial repair. Such combined modalities may facilitate myocardial regeneration by inducing endogenous cardiac cells to migrate, differentiate, and proliferate in situ, replacing lost endothelial cells and cardiomyocytes (Urbaneket al., 2005). However, despite recent progress in applying the approaches of regenerative medicine to the treatment of such diseases, valid strategies aimed at repairing the infarcted heart and, in general, at treating end-organ ischemia continue to be elusive. Major obstacles are the difficulty in isolating and delivering stem cells that are specifically effective in myocardial repair, and in stimulating recruitment of endogenous stem cells to the ischemic tissue. To address these issues, there has been increasing focus on novel biotechnologies or pharmacological strategies to enhance the implantation of exogenous stem cells or to boost endogenous regeneration of myocardial tissue. By employing three fundamental "tools", namely stem cells, biomaterials and growth factors (GFs) (Lavik \& Langer, 2004; Mikos et al., 2006), such tissue engineering strategies may enhance the efficacy of stem cell therapy in several ways: by mobilizing endogenous stem/progenitor cells in vivo; by promoting cell proliferation and differentiation; and by augmenting cell engraftment and survival in the injured myocardium. In general, because of 
the short half-lives of GFs in the body and the necessity to deliver them to specific target sites, GF injections themselves do not always produce the anticipated therapeutic effect. At present, GF delivery in regenerative medicine basically relies upon two strategies: 1) delivery of the GF genes; 2) direct delivery of GFs by incorporating them into a vehicle. In the gene delivery approach, delivery of the GF gene may result in higher and more constant levels of protein produced, since the gene - rather than a degradable protein - is being delivered (Haastert \& Grothe, 2007). Two major problems are associated however with this approach: 1) the complexity of cloning and integrating the gene into the target cells; 2) safety and efficiency of transduction. At present, there are insufficient well-controlled long-term studies in the preclinical area to make any conclusive statements about the clinical suitability/efficacy of gene delivery in humans. If resolved, cell-mediated synthesis of GFs should be associated with more efficient targeting of receptors and, consequently, a more robust and predictable approach in ischemic tissue regeneration.

\section{Stem cell basics and selection}

Stem cells are a population of immature tissue precursor cells capable of self-renewal as well as of differentiation into a spectrum of different cell types in appropriate conditions. In general, they share the following characteristics: (1) a high capacity for self-renewal; (2) the potential for differentiation in multiple cell types; (3) the ability to be cultured ex vivo and used for tissue engineering; and (4) plasticity (transdifferentiation ability) (Vats et al., 2005). On the basis of their differentiating potential, stem cells can currently be classified into four categories: (1) totipotent, (2) pluripotent, (3) multipotent, and (4) oligopotent or monopotent. Totipotent stem cells have the potential to differentiate into cells of all three main germinal layers (the ectodermal, endodermal, and mesodermal) and embryo-derived tissues. Pluripotent stem cells have the ability to differentiate only into tissues derived from the ectoderm, endoderm and mesoderm. Multipotent stem cells can differentiate into tissuespecific progenitor cells within a given organ. For example, multipotent blood stem cell or hematopoietic stem cells can develop into red blood cells, white blood cells, or platelets. Oligopotent or monopotent stem cells can only give rise to one or few types of specialized cells. On the basis of their origin and biological properties, stem cells can also be classified as either (1) embryonic stem cells or (2) adult stem cells. Embryonic stem cells are derived from the inner layer mass of the blastocyst and can be harvested from three sources: aborted fetuses (cadaveric stem cells), embryos left over from in vitro fertilization (discarded embryos), and embryos created in the laboratory solely for the purpose of producing stem cells (research embryos). In vitro differentiation of human embryonic stem cells into cardiomyocytes has been demonstrated by Kehat et al. (Kehat et al., 2001). However, ethical issues have been raised against harvesting human embryonic stem cells, especially if this process requires destruction of an embryo. Other potential obstacles to using embryonic stem cells are that recipients often need to receive immunosuppressants, because embryonic stem cells are potentially allogenic and strongly immunogenic. Uncontrolled differentiation of embryonic stem cells may cause other problems, such as the development of cardiac or vascular neoplasm. Transplanted embryonic stem cells may form teratomas if some undifferentiated totipotent cells are still present. The formation of teratomas -i.e., tumors containing a mix of differentiated human cell types, including cells characteristic of the ectoderm, mesoderm, and endoderm-in severe combined immunodeficiency (SCID) mice after injection with human embryonic stem cells has been observed (Thomson et al., 1998). 
Adult stem cells are the undifferentiated cells that exist in a differentiated tissue or organ and that are capable of specializing into cells of the tissue or organ from which they originated. Their capacity for self-renewal allows tissues and organs to maintain functional stability. Sources of adult stem cells include not only regenerating tissues, such as the heart, adipose tissue, bone marrow, blood, liver and epidermis, but also non-divisive tissues, such as the brain. Compared with embryonic stem cells, autologous adult stem cells are not faced with any major ethical or immunological controversies surrounding their use in the same individual from whom they were obtained. However, their ability to proliferate and differentiate is less powerful than that of embryonic stem cells; they are often difficult to identify, isolate, and purify; and they are not numerous enough for use in transplantation without being expanded in vitro substantially. For example, there is only one hematopoietic cell for every 1000-5000 bone marrow stromal cells (BMSCs). Adult stem cells do not replicate indefinitely in culture (Jiang et al., 2002), and they are referred to as multipotent or oligopotent stem cells. The partially differentiated cells are precursor or progenitor cells, which are characterized by the ability to proliferate. For instance, circulating endothelial progenitor cells (EPCs) can promptly differentiate into mature endothelial cells that replace dead or dying cells when the endothelium of arteries is injured by atherosclerosis (Urbich \& Dimmeler, 2004).

Selection of a suitable type of stem cells is a key issue for the success of stem cell therapy. Stem/progenitor cells used for transplantation should have the following characteristics: (1) high rates of survival and proliferation; (2) high capability of differentiation; and (3) the potential for engraftment and integration with native or host cardiac cells. Currently, both embryonic and adult stem cells are used in experimental cardiac cell transplantation studies, while only adult stem cells (e.g., bone marrow-derived mesenchymal cells, skeletal myoblasts, endothelial progenitor cells) are used in clinical trials. Each stem cell type has unique biological properties that offer both advantages and limitations to their use. Therefore, selection of the most suitable stem cells for use in ischemic patients is still a major focus of current research. The skeletal myoblasts or satellite cells are precursor cells of human skeletal muscle that originate from muscle stem cells (Angelis et al., 1999). They normally lie in a quiescent state under the basal membrane of muscular fibers, and have the potential for reentry into the cell cycle in response to injury, where they can divide and differentiate into functional muscle cells. Skeletal myoblasts can be obtained from individual patients themselves. Other theoretical advantages of using autologous skeletal myoblast are their rapid expansion in culture and their lower likelihood of tumor formation after transplantation. In addition, these cells have a chance of engrafting with native cardiomyocytes and surviving in infarcted regions of the heart since they are relatively resistant to ischemia. Animal studies have demonstrated that skeletal myoblasts can successfully accommodate themselves in the infarcted region of the heart, forming striated muscle fibers with intercalated discs in the host myocardium under the influence of factors in the cardiac environment (Murry et al., 1996). Improvement in systolic function has been noted after skeletal myoblast transplantation in ischemic and non-ischemic heart failure models (Atkins et al., 1999; Hagegeet al., 2001; Siminiak \& Kurpisz, 2003). Controversy still exists regarding the capability of stem cells to engraft and connect with native cardiomyocytes, despite promising results from preclinical studies (Suzuki et al., 2001; Menasche et al., 2003; Pagani et al., 2003; Smits et al., 2003). Bone marrow-derived stem cells are currently the most commonly used cells in cell transplantation therapy. The ideal stem 
cells from bone marrow for cardiac regeneration remain to be identified and many details remain to be elucidated. Yet, the clinical results from recent trials show the capability of these cells to ameliorate systo-diastolic heart function and decrease the size of the infarct region after intracoronary injection (Assmus et al., 2002; Strauer et al., 2002; Britten et al., 2003). Other studies (Perin et al., 2002; Perin et al., 2003) have demonstrated the safety and efficacy of transendocardial injection of autologous bone marrow mononuclear cells in patients with end-stage ischemic heart disease. Fetal cardiomyocytes still can enter the cell cycle and be expanded in culture. Successful cell transplantation using fetal cardiomyocytes was initially demonstrated in mice (Soonpaa et al., 1994; Li et al., 1995; Li et al., 1996), with findings in improvement of heart function and formation of new blood vessels in and around the cell graft area (Watanabe et al., 1998). These experiments show that fetal cardiomyocyte transplantation is feasible and potentially clinically relevant. Besides the ethical questions concerning the use of human fetal tissue, however, one limitation of using fetal cardiomyocytes is that lifelong immunosuppressive therapy may be necessary to prevent rejection. The optimal regimen and dose of immunosuppressive agents for cell transplantation would be here still unknown. A large number of studies have examined the differentiation of embryonic stem cells into cardiomyocytes, aiming at clarifying the mechanism of differentiation, identifying cell markers, and developing techniques for purifying embryonic stem cell-derived cardiomyocytes. The pluripotency of embryonic stem cells gives rise to their differentiation into more than 200 hundred kinds of cell lines. Protocols for the in vitro differentiation of embryonic stem cells into cardiomyocytes representing all specialized cell types of the heart, such as atrial-like, ventricular-like, sinus nodal-like, and Purkinje-like cells, have been established. Only $5-10 \%$ of these cells have been identified as cardiomyocytes. It may be ideal to separate cardiomyocytes from the undifferentiated ES cells before transplantation because of their potential to generate tumorlike tissue if implanted (Odorico et al., 2001). However, how to direct the differentiation of embryonic stem cells only into functional cardiomyocytes in vivo is still unclear. As stated above, another technical limitation is here the need for immunosuppressant therapy after transplantation. Endothelial progenitor cells can be identified in adult peripheral blood, bone marrow, and human umbilical cord blood.

Current experiments suggest that EPCs play an important role in vasculogenesis by differentiating into vascular endothelial cells, inhibiting ventricular remodeling through improvement in myocardial blood supply (Kamihata et al., 2001). Adipose tissue-derived stem cells may overcome major limitations in the use of adult stem cells harvested from essential organs such as muscle, skin, brain. liver and bone marrow. It has been shown that these cells can be induced to differentiate into multiple cell lineages, including adipose, cartilage and bone, muscle cells, neurons and endothelial cells (Zuk et al., 2001; Madonna et al., 2009). Injection of adipose tissue-derived stromal cells (ADSCs) has been recently shown to improve neovascularisation in the ischemic hind limb and the infarcted heart (see Madonna et al., 2009 for a general review of preclinical studies in the heart and hind limb ischemia models). Furthermore, recent studies (Puissant et al., 2005) have reported on the in vivo and in vitro immunosuppressive properties of ADSCs and their capability of escaping the immune surveillance. Therefore, major advantages of the adipose tissue as an alternative source of regenerative cells, include: 1 . yield: a therapeutic dose of regenerative cells can be isolated in approximately one hour without cell culture; 2 . safety: patients receive their own cells (autologous-use), with no risk of immune rejection or transmission; 3. versatility: stem 
cells from the adipose tissue benefit from multiple mechanisms of action. Recently it has been documented that the adult heart contains a pool of small cells expressing stem cell markers [c-kit, multi drug resistance 1 (MDR-1), and stem cell antigen 1 (Sca-1)]. Such cells also harbor telomerase activity, which is only present in replicating cells. These cardiac stem-progenitor cells (CSCs) not only can replenish the cardiomyocyte population, but are also able to regenerate coronary vessels (Beltrami et al., 2001). The actual number of CSCs remains controversial. Quantitative data in mouse, rat, dog, and human hearts have demonstrated that there is 1 CSC per approximately 30,000 to 40,000 myocardial cells: approximately $65 \%$ of all CSCs possess the 3 above-referred antigens (c-kit, MDR-1, and Sca1-like); approximately $20 \%$ possess 2 , and approximately $15 \%$ possess only 1 . Approximately $5 \%$ each of all CSCs exclusively express c-kit, MDR-1, or Sca-1. Furthermore, more premature resident CSCs, such as cardiospheres and isl1 $1^{+}$cardioblasts, have been recently identified (Messina et al., 2004). This variability in the number and type of CSCs reflects cells with distinct functional capacity and different stage of differentiation. In vivo experiments have shown that injected cardiospheres can produce functional improvement of the heart, with $37 \%$ improvement in fractional shortening in the injected group (Messina et al., 2004; Smith et al., 2007; Chimenti et al., 2010). Main advantages of CSCs are the following: 1. CSCs are autologous, and thus unlikely to trigger infectious or immunological complications; 2. CSCs are more cardiogenic than other adult stem cells; 3. CSCs trigger robust angiogenic responses after myocardial transplantation.

\section{Growth factor selection and vehicle-based delivery approach}

Vehicle-based delivery systems for growth factors, derived from diverse biomaterials, are used to increase their retention at treatment sites for a sufficient period of time to allow tissue regenerating cells to migrate into the area of injury, to proliferate and to differentiate, as well as to reduce the loss of bioactivity. They are also used to control toxicity induced by high concentrations of growth factors (Vasita \& Katti, 2006; Chou \& Leong, 2007). A major challenge inherent in these strategies is to identify growth factors and signaling pathways that selectively promote proliferation, migration, engraftment, and differentiation of resident CSCs or exogenous multipotent stem cells. This challenge relates also to the understanding of cellular uptake mechanisms, cell response to the mechanochemical microenvironment, the potential therapeutic utility of delivered biomolecules, and the exact requirements for multiple signals to drive the ischemic cardiovascular tissue regeneration process to completion. Current knowledge suggests that "cocktails" of biomolecules, or even cocktails of different types of stem cells, should be delivered locally, with specific and distinct pharmacokinetics/pharmacodynamics (e.g., the capacity of each single component of the cocktail to act distinctly in differentiating or in homing different endogenous stem cell/progenitor population), in order to mimic, as far as possible, the different requirements of the ischemic tissue during the various regeneration phases. Engineering natural and/or synthetic scaffolds to release several growth factors (or growth factor-encoding genes) in a sequential manner might affect more than one phase of the ischemic tissue healing process, for example, neovascularization and myogenesis, ultimately leading to cardiovascular tissue regeneration rather than repair. The growth factors that have been most intensively investigated in the regeneration of ischemic cardiovascular tissues include vascular endothelial growth factor (VEGF), transforming growth factor (TGF)- $\beta$, platelet-derived growth factors (PDGF, including PDGF-BB and PDGF-AA), insulin-like growth factor (IGF)- 
1, basic fibroblast growth factor (b-FGF) (Beohar et al., 2010). Overall, results indicate that these growth factors elicit significant proliferative and angiogenic effects. It is now well established that the utilization of multiple growth factors, rather than one, capable to act in a concentration- and time-dependent manner, is essential in the processes involved in the regeneration of ischemic cardiovascular tissues (Chen et al., 2010). An example is given by the need of having VEGF and PDGF together in order to promote angiogenesis. Indeed, VEGF is an initiator of angiogenesis, while PDGF promotes blood vessel maturation. As an example for this, a polymer scaffold constructed from Poly (Lactide-co Glycolide) (PLG), capable of delivering VEGF and PDGF together, with better results in terms of formation of mature vessels compared with the delivery of VEGF or PDGF singularly, has been produced (Richardson et al., 2001). In the selection of growth factors potentially capable to boost the regeneration of ischemic cardiovascular tissue, we and others have investigated on hepatocyte growth factor (HGF) and its receptor receptor mesenchymal-epithelial transition factor (Met). Ligand-receptor systems such HGF and its receptor, the tyrosine kinase Met, are potential candidates for therapeutic angiogenesis and for boosting migration, engraftment and commitment of CSCs because they promote the translocation of CSCs into the injured area, activate their growth and differentiation, and stimulate endothelial cell migration (Rappolee et al., 1996; Forte et al., 2006; Madonna et al., 2010). The strategy of combining stem cells, either native or gene-engineered to overexpress growth factors, with biopolymers that are functionalized with growth factors such as HGF, would facilitate myocardial regeneration: a) by supplying exogenous stem cells or GFs that stimulate resident CSC migration, engraftment and commitment to cardiomyocytes, and that induce and modulate arterial responses to ischemia; b) by supporting the maintenance of GFs and transplanted stem cells in the damaged tissues through the use of biocompatible and biodegradable polymers for a period of time sufficient to allow histological and anatomical restoration of the damaged tissue. These polymers can provide vehicles to deliver bioactive factors and stem cells into the infarcted heart or ischemic cardiovascular tissues. Finally, this approach would promote the ability of resident CSCs or of exogenous multipotent stem cells, such as adipose tissue-derived mesenchymal stem cells (AT-MSCs), to induce the healing of damaged tissue, by recruiting and directing these cells into the damaged area, by improving angiogenesis and, finally, by promoting the reperfusion of ischemic tissues.

\section{Growth factor-delivery systems and devices in the treatment of tissue ischemia}

Many limitations of stem cell therapies could be resolved by stimulating specific cellular functions for cell populations that normally are quiescent in the adult heart or that are not capable of replacing the dying cells. Compounds (cytokines and growth factors) that are simply injected into the lesions quickly disappear from the site of injection because they are removed by the blood flow and degraded by specific enzymes located in the extracellular microenvironment. To overcome this drawback, a possible strategy is to install a polymer functionalized with growth factors and stem cells into the damaged heart to stimulate the natural process of cardiac repair. Polymers tested in the past for their ability to support transplanted cells, without any conjugation with functional molecules, have been marginally effective. More novel polymers are conjugated with functional molecules (growth factors, chemotactic factors, cytokines), and are capable of stimulating specific normally quiescent cellular functions (Tatard et al., 2005). They are known as "smart" polymers because they 
"persuade" and guide the regenerative process, and are "biomimetics" because they use strategies occurring during the physiological regenerative process. Unlike the previous ones, novel polymers are "smart", in that they can acquire several biological functions depending on the bioactive factor or stem cell type to which they are conjugated. These polymers can prolong and amplify specific stem cell functions. They can stimulate the recruitment of circulating and resident stem cells and subsequently promote their adhesion to the damaged area, and can enhance survival, proliferation, and differentiation of stem cells into cardiac and vascular cells (Madonna \& De Caterina, 2009). Several of these features are critical to tissue regeneration, including restoration of the delivery of factors, nutrients, oxygen, and blood to necrotic tissues. To accomplish this, polymers must be functionalized by conjugation with bioactive factors. To date, delivery systems of growth factors are basically classified as: 1) reservoir systems; 2) environmentally responsive systems. Reservoir systems are one of the oldest methods used successfully to deliver drugs. The primary drug release mechanism from reservoir systems is diffusion-controlled release, characterized by an initial "burst release" phase followed by a phase of slower drug release from the carrier (Langer, 1983). Examples are hydrophilic matrices, that degrade when water enters and in this way release the drug (Franssen et al., 1999). Several reservoir systems have been developed to more closely control the release kinetics and avoid "burst type" release of the encapsulated factor(s). For example, the in vitro release of TGF- $\beta$ from novel, injectable hydrogels based on the polymer oligo(poly(ethylene glycol) fumarate) (OPF) has been investigated (Holland et al., 2003). This system has been extended to enable the dual delivery of IGF- 1 and TGF- $\beta$ by loading the two growth factors into either the OPF hydrogel phase or the gelatin microparticle phase of composites. Release profiles were successfully manipulated by altering the phase of growth factor loading and the extent of microparticle cross linking (Holland et al., 2005).

Environmentally responsive systems are able to match a patient's physiological needs at the appropriate time and/or the correct site. They are able to deliver a certain amount of growth factor(s) in response to a biological state (Qiu \& Park, 2001). They are constituted by sensitive hydrogels that can control the release of drugs by changing the gel structure according to environmental stimulation, such as temperature, $\mathrm{pH}$, and/or ion concentration. Temperature-sensitive hydrogels are able to swell or shrink as the temperature of the surrounding fluid varies (Ramanan et al., 2006). The poly-N-isopropylacrylamide (PNIPAAm) hydrogel is a typical example of temperature-sensitive hydrogel, featuring solto-gel transition at a critical solution temperature of about $35^{\circ} \mathrm{C}$. This polymer releases the drug with the transition from gel to sol (Zhang et al., 2004), and is of particular interest in those clinical situations, such as tissue ischemia, characterized by low tissue temperature. Similarly, pH-sensitive polymers contain pendant acidic (e.g., carboxylic and sulphonic acids) or basic (e.g., ammonium salts) groups that either accept or release protons in response to changes in environmental $\mathrm{pH}$ (Qiu \& Park, 2001). Such polymers can release a drug when the environmental $\mathrm{pH}$ decrease. These acidic $\mathrm{pH}$-sensitive polymers may be useful for the treatment of tissue ischemia and inflammation (Matsusaki \& Akashi, 2005). A very recent work has also shown the capability of new magnetic particles embedded in polymer gels, termed ferrogels, to release drugs in response to magnetic fields (Zhao et al., 2011). Here the authors created alginate-based porous scaffolds containing the arginineglycine-aspartic acid amino acid sequence, covalently coupled with the alginate and embedded with $10 \mathrm{~nm}$ iron oxide particles. Under applied magnetic fields this superparamagnetic gel undergoes prompt deformation, causing water flow through the 
interconnected pores, thus triggering the release of biological agents. The authors here showed the capability of these ferrogels to promptly release several drugs, including mitoxantrone, plasmid DNA, chemokines, as well as cells under the control of external magnetic fields in vitro and in vivo (Zhao et al., 2011). In our laboratory, we have been working on a novel and still poorly investigated strategy using polymeric pharmacologically active microspheres (PAM), 50-100 $\mu \mathrm{m}$ in diameter, with an in vivo halflife of 1 month, that encapsulate bioactive factors gradually released into the injection area (Tatard et al., 2005; Madonna \& De Caterina, 2009), and providing a vehicle for stem cell, drug, growth factors and gene delivery (Fig. 1). The chemical composition of these polymers is based on FDA-approved compounds, namely, polylactic acid (PLA), poly-D,L-lactic-coglycolic (PLGA), and polycaprolactone (PCL) (Mudargi et al., 2008). PAM also satisfy the need for resistance to accelerated degradation that may happen in the harsh microenvironment of tissue ischemia. In addition, compared with other polymers, PLGA has more hydrophilic domains, which favors cell attachment, and therefore constitute a good support material for bioactive molecules that mobilize and home circulating progenitor/stem cells to the injured area (Tatard et al., 2005).

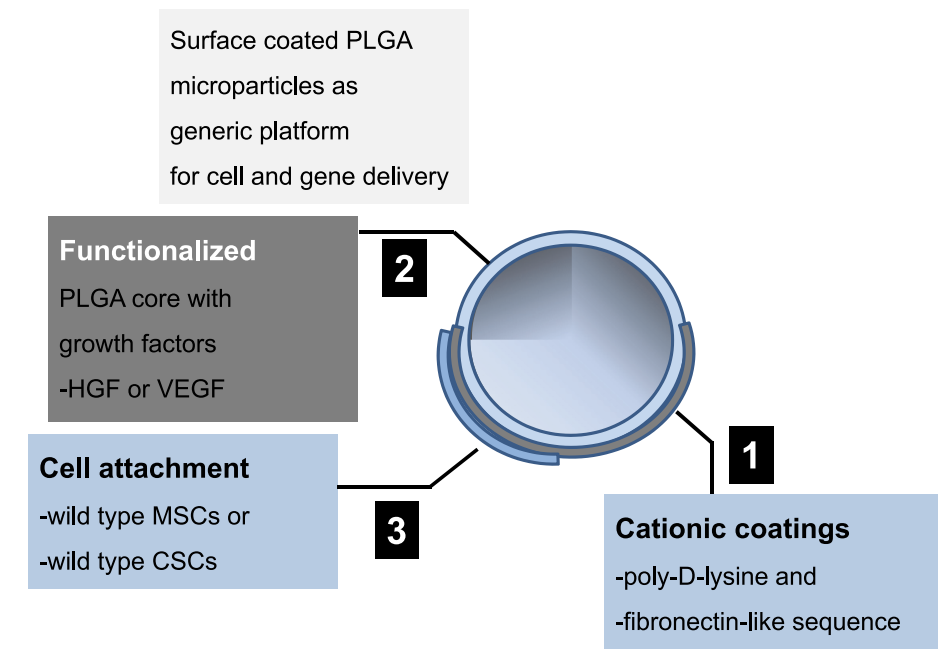

Fig. 1. The concept of multifunctional pharmacologically active microspheres (PAM). Surface-coated PLGA microparticles form a generic platform that can be functionalized with drugs, growth factors or genes and externally coated with stem cells. Abbreviations: PLGA, poly-D,L-lactic-co-glycolic; HGF, hepatocyte growth factor; VEGF, vascular endothelial growth factors; MSCs, mesenchymal stem cells; CSCs, cardiac stem cells.

\section{The gene delivery approach}

Beside the incorporation into a carrier vehicle, different approaches and strategies for direct delivery of growth factors have been employed, including the delivery of growth factor genes. In order to mimic the natural healing process of the tissue successfully, this strategy mostly requires a localized application of multiple, rather than one, genes that encode for and activate the synthesis of sequential multiple growth factors with synergistic effects on 
tissue regeneration (De Laporte et al., 2009; Donofrio et al., 2010; Fujii et al., 2011; Liu et al., 2011). However, low transfection efficiencies, inefficient gene targeting, low gene expression levels, and undesired gene integration into host DNA are all challenges that may undermine growth factor gene delivery as a better approach instead of the growth factor protein delivery (Juillerat-Jeanneret \& Schmitt, 2007). Gene delivery can be performed either by directly introducing the delivery vector into the anatomical site (in vivo) or by harvesting cells from the patient, transferring the gene(s) to the cells in tissue culture and then transferring the genetically modified cells back into the patient (ex vivo). In another recent review article we have specifically discussed cell-mediated HGF/Met gene transfer for myocardial regeneration (Madonna et al., 2010). A more general overview of preclinical studies using in vivo and ex vivo gene delivery strategies is provided here.

\section{In vivo approaches}

An in vivo gene delivery approach can be accomplished either by direct injection of viral vectors or transfection reagents into the site, or by attaching the delivery vector to the scaffold (Bleiziffer et al., 2007). This latter approach is more straightforward, but is limited by inefficient gene delivery and nonspecific cellular targeting. An early evaluation of in vivo gene delivery for therapeutic angiogenesis involved the direct intramyocardial injection of VEGF DNA using an adenovirus vector in patients with otherwise inoperable coronary artery disease and intractable angina pectoris (Rosengart et al., 1999). Phase I clinical trials documented the safety and feasibility, but not clear-cut clinically relevant efficacy of gene transfer using plasmid DNA, most likely because of the low levels of growth factor achieved with single injections of plasmid DNA (Rosengart et al., 1999). As of today, most clinical trials have failed to show any benefit of VEGF in vivo gene therapy, even by using different routes of intramyocardial administration that can achieve the transfer of high doses of the specific DNA, such as with percutaneous guidance catheter systems. The double-blind, placebo-controlled NOGA angiogenesis Revascularization Therapy: assessment by RadioNuclide Imaging (NORTHERN) study showed no benefit of plasmid DNA-VEGF gene therapy at 3 and 6 months in terms of change in myocardial perfusion from baseline in patients with refractory Canadian Cardiovasculart Society (CCS) class 3 angina (Stewart et al., 2009).

\section{Ex vivo approaches}

Although traditional delivery of cells associated with growth factors is still a candidate strategy in laboratory-based trials, the most frequently investigated cell transplantation in tissue engineering to date is cell-based gene therapy. This therapy typically relies on transplanting cells, such as stem cells, lymphocytes, fibroblasts, or - alternatively - the cells of interest, that are removed from the body and injected after therapeutic transgene modifications (Fischer et al., 2009; Cho \& Marban, 2010; Madonna et al., 2010). This ex vivo approach allows for targeting of specific cells for gene delivery, supplies cells that may directly participate in the regenerative process, allows for both autocrine and paracrine effects from the expressed growth factor, and avoids the safety risks of directly injecting viral vectors or transfection reagents in vivo. This approach, however, involves an extra step to manipulate and expand cells in tissue culture, and has the risk of contamination. Additionally, the ex vivo approach does not eliminate the possibility of retroviral vectors causing insertional activation of other genes, the over-expression of which may cause cancer, as experienced when using ex vivo gene therapy for the treatment of children with Xlinked severe combined immune deficiency (Gansbacher \& European Society of Gene 
Therapy, 2003). Progress in the field of gene therapy has been limited by safety concerns related to delivery vectors. Genetically modified cells are potentially able to provide a stable source of growth factors at a level that is sufficient to elicit a biological response. Autologous cells may also be used in this approach via the isolation of a small number of differentiated adult cells or stem cells, followed by in vitro expansion to produce an appropriate supply. The cells may naturally secrete or be genetically modified in vitro to overexpress the factor, either transiently or permanently. After their genetic modification, the cells are allowed to grow in vitro and increase in number, so as to synthesize and secrete the desired growth factors at the site where they have been transplanted. This approach may be particularly appropriate for delivery of growth factors that act by paracrine or juxtacrine mechanisms.

\section{Lentiviral and non-lentiviral vectors for gene delivery into stem cells}

The introduction of growth factor genes in stem cells can be performed by using viral or non-viral vectors. In the choice of using viral vectors, important experimental variables for a successful gene therapy include the multiplicity of infection (MOI), time length for viral incubation and medium used for viral incubation. An optimal combination of such experimental conditions would increase gene transfer efficiency and possibly obviate the need for selective antibiotic-based enrichment and long-term culture, which may contribute to senescence or compromise the long-term engraftment efficiency and/or multipotency of grafted cells (Rombouts \& Ploemacher, 2003). In addition, by increasing gene transfer efficiency, fewer cells may be required to achieve a therapeutic effect. This justifies the use of lentiviral vectors for transducing adult stem cells, by virtue of their ability to transduce both dividing and non-dividing cells and their relative ease of use and comparable nature to adeno-associated viral (AAV) vectors, which are clinically preferred. For the transduction of adult stem cells, lentivirus-based systems are virtually ideal, since they overcome most problems, including the short duration of gene expression and the occurrence of significant inflammatory responses, which plague other types of gene vectors (such as adenoviruses). Lentiviruses are a subgroup of retroviruses that include the human type 1 immunodeficiency virus (HIV). While retroviral systems are inefficient in transducing nondividing or slowly dividing cells, lentivirus-based vectors, after being pseudotyped with vesicular stomatitis virus glycoprotein G (VSV-G) (i.e., using the glycoprotein envelope from the vesicular stomatitis virus to package recombinant retroviruses) (Emi et al., 1991), can mediate genome integration into both non-dividing and dividing cells (Fig. 2). There is evidence that lentiviral vectors can also transduce more primitive, quiescent progenitors with stable transgene integration (Case et al., 1999). In comparison with other retroviral vectors, lentiviral systems allow the immediate transduction without prior expansion, or with growth factor stimulation for only short exposure times. Compared with adenoviral vectors, lentiviral vectors also offer the major advantages of causing little or no disruption of the target cells and of not promoting any inflammatory response (Lever, 1996). AAV vectors represent an alternate type of vector that may also be used for long-term transgene expression in the heart through cell-based therapy (Svensson et al., 1999). Like lentiviruses, AAV can stably integrate into the host genome providing long-term transgene expression, with a minimum inflammatory response. However, AAV can cause insertional mutagenesis and can only carry genes which are less than $5 \mathrm{~kb}$ (Donsante et al., 2007). A possible drawback of the use of lentiviral and AAV vectors for delivering genes that encode for 
growth factors might be that they can cause a chronic overexpression of the protein, with an uncertain therapeutic effect. Short-term gene expression of the growth factor gene would be desirable if the goal is to deliver a secreted protein, such as insulin-like growth factor-1 (IGF-1), vascular endothelial growth factor (VEGF) and HGF, while long-term expression would be preferable if the goal is to express membrane proteins such as receptors for growth factors that require stable expression. Possible strategies to induce short-term gene expression of the transgene include plasmid transfection or the use of adenoviral vectors (Rabbany et al., 2009). Limitations of these strategies are the low transfection efficiency with plasmids and the immunogenic response of the host with adenoviruses.

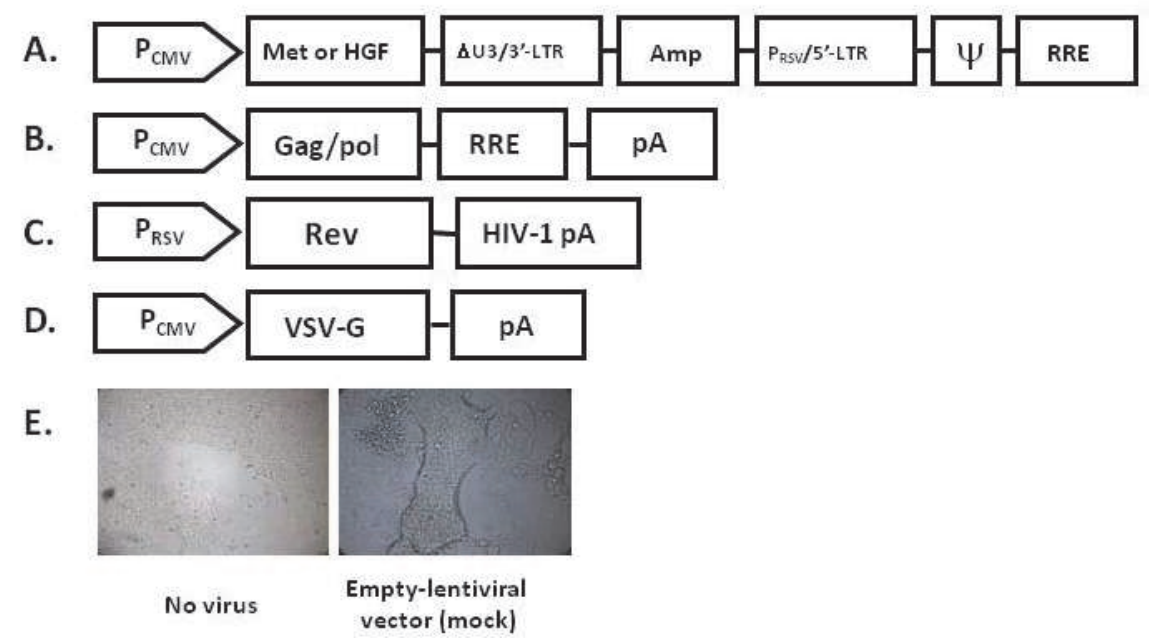

Fig. 2. Schematic representation of the transducing vector construct (a), packaging construct (b, c) and VSV-G Env-expressing construct (d). Abbreviations: Amp: ampicillin resistance gene; $\Delta \mathrm{U} 3$ : deleted region of the 3'LTR, which allows for biosafety of the vector; Gag, pol, env: genes codifying for envelope proteins; PCMV: cytomegalovirus promoter; 3'-LTR: 3'long terminal repeats for viral packaging; PRSV: Rous Sarcoma Virus (RSV) enhancer/ promoter; 5'-LTR: 5'-long terminal repeats for viral packaging; w psi: packaging sequence for viral packaging; pA: polyadenylation signal; RRE: HIV-1 Rev response element; VSV-G: G glycoprotein gene from Vesicular Stomatitis Virus (VSV-G) as a pseudotyping envelope. (e) Photographs representing the experimental steps of production of the lentiviral stock (containing the packaged expression construct) by cotransfecting the packaging construct (b, c), the VSV-G Env-expressing construct (d) and transducing vector construct (a) into the 293FT virus-producing cell line. The expression of the VSV-G glycoprotein causes 293FT cells to fuse, resulting in the appearance of large, multinucleated cells known as syncytia.

\section{Conclusions}

Growth factor delivery and tissue engineering have emerged as new concepts that focus on tissue regeneration from cells with the support of biomaterials and growth factors 
(Ikada, 2006). Various delivery methods are available to administer growth factors to ischemic tissues. Among these strategies, the cell-based delivery of growth factor genes is of great potential interest, since the in situ expression of the growth factor gene may result in higher and more constant levels of protein production. With respect to the delivery of growth factors, a major challenge is to identify growth factors and signaling pathways that selectively promote proliferation, migration, engraftment and differentiation of resident CSCs or exogenous multipotent stem cells. Current knowledge suggests that "cocktails" of biomolecules (growth factors or DNA), or even more cocktails of exogenous stem/committed progenitor cells (myogenic or angiogenic cells) with a wide spectrum of differentiation capabilities, should be delivered locally, in order to mimic, as far as possible, the different requirements of the ischemic tissue during the different regeneration phases. To deliver multiple growth factor genes and stem cells with distinct release and dynamic profiles, a greater understanding of the requirements for regenerating complex and functional tissues such as the myocardium is necessary. Challenges in this area revolve on controlling reciprocal interactions among those cells and signals. Winning these challenges would open a new era in stem cell and gene therapy research for both laboratory-based scientists and practicing physicians. From a bedside point of view, preclinical studies in large animals (such as porcine model) are required to carefully evaluate potential human therapies.

\section{Acknowledgements}

This work was supported by grants from the Italian Ministry of University and Scientific Research and from the Istituto Nazionale Ricerche Cardiovascolari (INRC).

The Authors declare no conflict of interest as related to the topics here discussed.

\section{References}

Angelis, D., L. Berghella, et al. (1999). "Skeletal myogenic progenitors originating from embryonic dorsal aorta coexpress endothelial and myogenic markers and contribute to postnatal muscle growth and regeneration." J Cell Biol 147: 869-77.

Assmus, B., V. Schachinger, et al. (2002). "Transplantation of progenitor cells and regeneration enhancement in acute myocardial infarction (TOPCARE-AMI)." Circulation 106: 3009-17.

Atkins, B. Z., M. T. Hueman, et al. (1999). "Myogenic cell transplantation improves in vivo regional performance in infracted rabbit myocardium." J Heart Lung Transplant 18: 1173-80.

Beltrami, A. P., K. Urbanek, et al. (2001). "Evidence that human cardiac myocytes divide after myocardial infarction." N Engl J Med 344: 1750-1757.

Beohar, N., J. Rapp, et al. (2010). "Rebuilding the damaged heart: the potential of cytokines and growth factors in the treatment of ischemic heart disease." J Am Coll Cardiol 56: 1287-1297.

Bleiziffer, O., E. Eriksson, et al. (2007). "Gene transfer strategies in tissue engineering." J Cell Mol Med 11: 206-223. 
Britten, M. B., N. D. Abolmaali, et al. (2003). "Infarct remodel1ing after intracoronary progenitor cell treatment in patients with acute myocardial infarction (TOPCAREAMI)." Circulation 108: 2212-8.

Camarata, P. J., R. Suryanarayanan, et al. (1992). "Sustained release of nerve growth factor from biodegradable polymer microspheres." Neurosurgery 30: 313-9.

Case, S. S., M. A. Price, et al. (1999). "Stable transduction of quiescent CD34(+)CD38(-) human hematopoietic cells by HIV-1-based lentiviral vectors." Proc Natl Acad Sci U $S$ A 96(6): 2988-93.

Chen, F. M., M. Zhang, et al. (2010). "Toward delivery of multiple growth factors in tissue engineering." Biomaterials 31: 6279-6308.

Chimenti, I., R. R. Smith, et al. (2010). "Relative roles of direct regeneration versus paracrine effects of human cardiosphere-derived cells transplanted into infarcted mice." Circ Res 106(5): 971-80.

Cho, H. C. and E. Marban (2010). "Biological therapies for cardiac arrhythmias: can genes and cells replace drugs and devices?" Circ Res 106: 674-685.

Chou, C. and K. W. Leong (2007). "Biomaterials approach to expand and direct differentiation of stem cells." Mol Therapy 15: 467-480.

De Laporte, L., Y. Yang, et al. (2009). "Plasmid releasing multiple channel bridges for transgene expression after spinal cord." Mol Therapy 17: 318-326.

Donofrio, G., A. Capocefalo, et al. (2010). "Virally and physically transgenized equine adipose-derived stromal cells as a cargo for paracrine secreted factors." BMC Cell Biol 23: 11-73.

Donsante, A., D. G. Miller, et al. (2007). "AAV vector integration sites in mouse hepatocellular carcinoma." Science 317(5837): 477.

Emi, N., T. Friedmann, et al. (1991). "Pseudotype formation of murine leukemia virus with the G protein of vesicular stomatitis virus." J Virol 65: 1202-1207.

Fischer, K. M., C. T. Cottage, et al. (2009). "Enhancement of myocardial regeneration through genetic engineering of cardiac progenitor cells expressing Pim-1 kinase." Circulation 120: 2077-2087.

Forte, G., M. Minieri, et al. (2006). "Hepatocyte growth factor effects on mesenchymal stem cells: proliferation, migration, and differentiation." Stem Cells 24(1): 23-33.

Franssen, O., R. D. van Ooijen, et al. (1999). "Enzymatic degradation of cross-linked dextrans." Macromolecules 32: 2896-2902.

Fujii, H., S. H. Li, et al. (2011). "Repeated and targeted transfer of angiogenic plasmids into the infarcted rat heart via ultrasound targeted microbubble destruction enhances cardiac repair." Eur Heart J Jan 8.

Gansbacher, B. and European Society of Gene Therapy (2003). "Report of a second serious adverse event in a clinical trial of gene therapy for X-linked severe combined immune deficiency (X-SCID). Position of the European Society of Gene Therapy (ESGT)." J Gene Med 5: 261-262.

Haastert, K. and C. Grothe (2007). "Cardiovascular gene therapy: current status and therapeutic potential." Br J Pharmacol 152: 175-188. 
Hagege, A. A., J. T. Vilquin, et al. (2001). "Regeneration of the myocardium: a new role in the treatment of ischemic heart disease? ." Hypertension 38: 1413-5.

Hirsch, A. T., Z. J. Haskal, et al. (2006). "ACC/AHA 2005 practice guidelines for the menagement of patients with peripheral arterial disease (lower extremity, renal, mesenteric, and abdominal aortic): a collaborative report from the American Association for Vascular Surgery/Society for Vacular Surgery, Society for Cardiovascular Abgiography and Interventions, Society for Vascular Medicine and Biology, Society of Interventional Radiology, and the ACC/AHA Task Force on Practice Guidelines (Writing Committee to Develop Guidelines for the Menagement of Patients with Peripheral Arterial Disease): endorsed by the American Association of Cardiovascular and Pulmonary Rehabilitation; National Heart, Lung, and Blood Institute; Society for Vascular Nursing; TransAtlantic Inter-Society Consensus; and Vascular Disease Foundation." Circulation 113: e463.

Holland, T. A., Y. Tabata, et al. (2003). " In vitro release of transforming growth factor-beta 1 from gelatin microparticles encapsulated in biodegradable, injectable oligo(poly(ethylene glycol) fumarate) hydrogels." J Control Release 91: 299-313.

Holland, T. A., Y. Tabata, et al. (2005). "Dual growth factor delivery from degradable oligo(poly( ethylene glycol) fumarate) hydrogel scaffolds for cartilage tissue engineering." J Control Release 101: 111-125.

Ikada, Y. (2006). "Challenges in tissue engineering." J R Soc Interface 3: 589-601.

Jiang, Y. H., B. N. Jahagirdar, et al. (2002). "Pluripotency of mesenchymal stem cells derived from adult marrow." Nature 418: 41-9.

Jollivet, C., A. Aubert-Pouessel, et al. (2004). "Striatal implantation of GDNF releasing biodegradable microspheres promotes recovery of motor function in a partial model of Parkinson's disease." Biomaterials 25: 933-42.

Juillerat-Jeanneret, L. and F. Schmitt (2007). "Chemical modification of therapeutic drugs or drug vector systems to achieve targeted therapy: looking for the grail." Med Res Rev 27: 574-590.

Kamihata, H., H. Matsubara, et al. (2001). " Implantation of bone marrow mononuclear cells into ischemic myocardium enhances collateral perfusion and regional function via side supply of angioblasts, angiogenic ligands, and cytokines." Circulation 104: 1046-52.

Kehat, I., D. Kenyagin-Karsenti, et al. (2001). "Human embryonic stem cells can differentiate into myocytes with structural and functional properties of cardiomyocytes." J Clin Invest 108: 407-414.

Krewson, C. E. and W. M. Saltzman (1996). "Transport and elimination of recombinant human NGF during long-term delivery to the brain." Brain Res 727: 169-81.

Langer, R. (1983). "Implantable controlled release systems." Pharmacol Ther 21: 35-51.

Lavik, E. and R. Langer (2004). "Tissue engineering: current state and perspectives." Appl. Microbiol. Biotechnol 65: 1-8.

Lever, A. M. (1996). "HIV and other lentivirus-based vectors." Gene Ther 3: 470-471. 
Li, R. K., Z. Q. Jia, et al. (1995). "Cardiomyocyte transplantation improves heart function." Ann Thorac Surg 62: 654-661.

Li, R. K., D. A. Mickle, et al. (1996). " In vivo survival and function of transplanted rat cardiomyocytes." Circ Res 78: 283-288.

Liu, J., L. Xu, et al. (2011). "Temporally controlled multiple-gene delivery in scaffolds: a promising strategy to enhance bone regeneration." Med Hypothesis 76: 173-5.

Madonna, R. and R. De Caterina (2008). "In vitro neovasculogenic potential of resident adipose tissue precursors." Am J Physiol - Cell Physiol 295 (5): c1271-80.

Madonna, R. and R. De Caterina (2009). "Enhancement of the regenerative capacity of stem cells by the "Pharmacologically Active Microcarriers": a biomimetic support for cells and a growth factor delivery vector." Acta from the V workshop of the Italian Society of Cardiovascular Research I: 20-25.

Madonna, R., Y. J. Geng, et al. (2009). "Adipose Tissue-Derived Stem Cells. Characterization and Potential for Cardiovascular Repair." Arterioscler Thromb Vasc Biol 29: 1723-9

Madonna, R., R. Rokosh, et al. (2010). "Hepatocyte Growth Factor/Met Gene Transfer in Cardiac Stem Cells - Potential for Cardiac Repair." Basic Card Res 105: 443-52

Madonna, R., J. T. Willerson, et al. (2008). "Myocardin a enhances telomerase activities in adipose tissue mesenchymal cells and embryonic stem cells undergoing cardiovascular myogenic differentiation." Stem Cells 26(1): 202-11.

Marban, E. (2007). "Big cells, little cells, stem cells: agents of cardiac plasticity." Circ Res 100: $445-446$.

Matsusaki, M. and M. Akashi (2005). "Novel fucntional biodegradable polymer IV: pHsensitive controlled release of fibroblast growth factor-2 from a poly(gammaglutamic acid)-sulfonate matrix for tissue engineering." Biomacromolecules 6(6): 3351-6.

Menasche, P., A. A. Hagege, et al. (2003). "Autologous skeletal myoblast transplantation for severe postinfarction left ventricular dysfunction." J Am Coll Cardiol 41: 1078-1083.

Menei, P., E. Jadaud, et al. (2004). "Stereotaxic implantation of 5- fluorouracil-releasing microspheres in malignant glioma." Cancer 100: 405-10.

Menei, P., J. M. Pean, et al. (2000). "Intracerebral implantation of NGF-releasing biodegradable microspheres protects striatum against excitotoxic damage." Exp Neurol 161: 259-72.

MERIT-HF Study Group (1999). "Effect of metoprolol CR/XL in chronic heart failure: Metoprolol CR/XL Randomised Intervention Trial in Congestive Heart Failure (MERIT-HF)." Lancet 353: 2001-2007.

Messina, E., L. De Angelis, et al. (2004). "Isolation and expansion of adult cardiac stem cells from human and murine heart." Circ Res 95: 911-921.

Mikos, A. G., S. W. Herring, et al. (2006). "Engineering complex tissues." Tissue Eng 12: 33073339.

Mittal, S., A. Cohen, et al. (1994). "In vitro effects of brain derived neurotrophic factor released from microspheres." Neuroreport 5: 2577-82. 
Mudargi, R. C., V. R. Babu, et al. (2008). "Nano/micro technologies for delivering macromolecular therapeutics using poly(D,L-lactide-co-glycolide) and its derivatives." J Control Release 125(3): 193-209.

Murry, C. E., R. W. Wlseman, et al. (1996). "Skeletal myoblast transplantation for repair of myocardial necrosis." J Clin Invest 98: 2512-23.

Nakagami, H., K. Maeda, et al. (2005). "Novel autologous cell therapy in ischemic limb disease through growth factor secretion by cultured adipose tissue-derived stromal cells." Arterioscler Thromb Vasc Biol 25(12): 2542-7.

Odorico, J. S., D. S. Kaufman, et al. (2001). "Multilineage differentiation from human embryonic stem cell lines." Stem Cells 19: 193-204.

Pagani, F. D., H. DerSimonian, et al. (2003). "Autologous skeletal myoblasts transplantated to ischemia-damaged myocardium in humans. Histological analysis of cell survival and differentiation." J Am Coll Cardiol 41: 879-88.

Pean, J. M., P. Menei, et al. (2000). "Intraseptal implantation of NGF-releasing microspheres promote the survival of axotomized cholinergic neurons." Biomaterials 21: 2097-101.

Perin, E. C., H. F. R. Dohmann, et al. (2003). "Transendocardial, autologous bone marrow cell transplantation for severe, chronic ischemic heart failure." Circulation 107: 2294-2302.

Perin, E. C., G. V. Silva, et al. (2002). "Assessing myocardial viability and infarct transmurality with left ventricular electromechanical mapping in patients with stable coronary artery disease: validation by delayed-enhancement magnetic resonance imaging." Circulation 106: 957-61.

Puissant, B., C. Barreau, et al. (2005). "Immunomodulatory effect of human adipose tissuederived adult stem cells: comparison with bone marrow mesenchymal stem cells." Br J Haematol 129: 118-129.

Qiu, Y. and K. Park (2001). "Environment-sensitive hydrogels for drug delivery." Adv Drug Deliv Rev 53: 321-339.

Rabbany, S. Y., J. Pastore, et al. (2009). "Continuous delivery of stromal cell-derived factor-1 from alginate scaffolds accelerates wound healing." Cell Transpl. doi:10.3727/096368909X481782.

Ramanan, R. M., P. Chellamuthu, et al. (2006). "Development of a temperature-sensitive composite hydrogel for drug delivery applications." Biotechnol Prog 22: 118-125.

Rappolee, D. A., A. Iyer, et al. (1996). "Hepatocyte growth factor and its receptor are expressed in cardiac myocytes during early cardiogenesis." Circ Res 78(6): 1028-36.

Rehman, J., D. Traktuev, et al. (2004). "Secretion of angiogenic and antiapoptotic factors by human adipose stromal cells." Circulation 109(10): 1292-8.

Richardson, T. P., M. C. Peters, et al. (2001). "Polymeric system for dual growth factor delivery." Nature Biotechnology 19: 1029-1034.

Rombouts, W. J. and R. E. Ploemacher (2003). "Primary murine MSC show highly efficient homing to the bone marrow but lose homing ability following culture." Leukemia 17: 160-170.

Rosengart, T. K., L. Y. Lee, et al. (1999). "Angiogensis gene therapy: phase I assessment of direct intramyocardial administration of an adenovirus vector expressing VEGF121 
cDNA to individuals with clinically significant severe coronary artery disease." Circulation 100: 468-74.

Siminiak, T. and M. Kurpisz (2003). "Myocardial replacement therapy." Circulation 108: 1167-71.

Smith, R. R., L. Barile, et al. (2007). "Regenerative potential of cardiosphere-derived cells expanded from percutaneous endomyocardial biopsy specimens." Circulation 115(7): 896-908.

Smits, P. C., R. J. van Geuns, et al. (2003). "Catheter-based intramyocardial injection of autologous skeletal myoblasts as a primary treatment of ischemic heart failure: clinical experience with six-month follow-up." J Am Coll Cardiol 42: 2063-9.

Soonpaa, M. H., G. Y. Koh, et al. (1994). "Formation of nascent intercalated disks between grafted fetal cardiomyocytes and host myocardium." Science 264: 98-101.

Stewart, D. J., M. J. Kutryk, et al. (2009). "VEGF gene therapy fails to improve perfusion of ischemic myocardium in patients with advanced coronary disease: results of the NORTHERN trial." Mol Therapy 17: 1109-15.

Strauer, B. E., M. Brehm, et al. (2002). "Repair of infracted myocardium by autologous intracoronary mononuclear bone marrow cell transplantation in humans." Circulation 106: 1913-8.

Suzuki, K., B. Murtuza, et al. (2001). "Cell transplantation for the treatment of acute myocardial infarction using vascular endothelial growth factor-expressing skeletal myoblasts." Circulation 104: I107-212.

Svensson, E. C., D. J. Marshall, et al. (1999). "Efficient and stable transduction of cardiomyocytes after intramyocardial injection or intracoronary perfusion with recombinant adeno-associated virus vectors." Circulation 99: 201-205.

Tatard, V. M., M. C. Venier-Julienne, et al. (2005). "Pharmacologically active microcarriers: a tool for cell therapy." Biomaterials 26(17): 3727-37.

Thomson, J. A., J. Itskovitz-Eldor, et al. (1998). "Embryonic stem cell lines derived from human blastocysts." Science 282: 1145-7.

Urbanek, K., M. Rota, et al. (2005). "Cardiac stem cells possess growth factor-receptor systems that after activation regenerate the infarcted myocardium, improving ventricular function and long-term survival." Circ Res 97(7): 663-73.

Urbich, C. and S. Dimmeler (2004). "Endothelial progenitor cells, characterization and role in vascular biology." Circ Res 95: 343-353.

Vasita, R. and D. S. Katti (2006). "Growth factor-delivery systems for tissue engoneering: a materials perspective." Expert Rev Med Devices 3: 29-47.

Vats, A., R. C. Bielby, et al. (2005). "Stem cells." Lancet 366: 592-602.

Watanabe, E., D. M. Smith, et al. (1998). "Cardiomyocyte transplantation in a porcine myocardial infarction model." Cell Transplant: 239-46.

Zhang, X., D. Wu, et al. (2004). "Synthesis and characterization of partially biodegradable, temperature and $\mathrm{pH}$ sensitive Dex-MA/PNIPAAm hydrogels." Biomaterials 25: 4719-4730.

Zhao, X., J. Kimb, et al. (2011). "Active scaffolds for on-demand drug and cell delivery." PNAS 108: 67-72. 
Zuk, P. A., M. Zhu, et al. (2001). "Multilineage cells from human adipose tissue: implications for cell-based therapies." Tissue Eng 7: 211-228. 


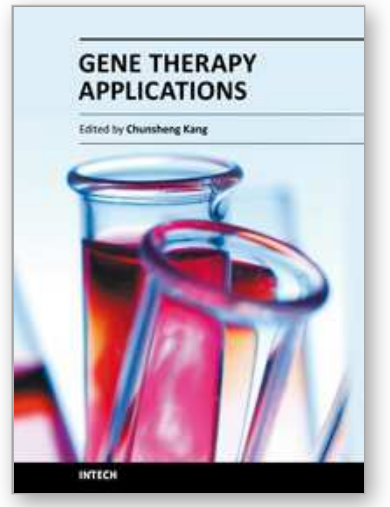

\author{
Gene Therapy Applications \\ Edited by Prof. Chunsheng Kang
}

ISBN 978-953-307-541-9

Hard cover, 492 pages

Publisher InTech

Published online 23, August, 2011

Published in print edition August, 2011

The aim of our book is to provide a detailed discussion of gene therapy application in human diseases. The book brings together major approaches: (1) Gene therapy in blood and vascular system, (2) Gene therapy in orthopedics, (3) Gene therapy in genitourinary system, (4) Gene therapy in other diseases. This source will make clinicians and researchers comfortable with the potential and problems of gene therapy application.

\title{
How to reference
}

In order to correctly reference this scholarly work, feel free to copy and paste the following:

Rosalinda Madonna and Raffaele De Caterina (2011). Cell-Based Gene Therapies and Stem Cells for Regeneration of Ischemic Tissues, Gene Therapy Applications, Prof. Chunsheng Kang (Ed.), ISBN: 978-953307-541-9, InTech, Available from: http://www.intechopen.com/books/gene-therapy-applications/cell-basedgene-therapies-and-stem-cells-for-regeneration-of-ischemic-tissues

\section{INTECH}

open science | open minds

\section{InTech Europe}

University Campus STeP Ri

Slavka Krautzeka 83/A

51000 Rijeka, Croatia

Phone: +385 (51) 770447

Fax: +385 (51) 686166

www.intechopen.com

\section{InTech China}

Unit 405, Office Block, Hotel Equatorial Shanghai

No.65, Yan An Road (West), Shanghai, 200040, China

中国上海市延安西路65号上海国际贵都大饭店办公楼 405 单元

Phone: +86-21-62489820

Fax: +86-21-62489821 
(C) 2011 The Author(s). Licensee IntechOpen. This chapter is distributed under the terms of the Creative Commons Attribution-NonCommercialShareAlike-3.0 License, which permits use, distribution and reproduction for non-commercial purposes, provided the original is properly cited and derivative works building on this content are distributed under the same license. 